
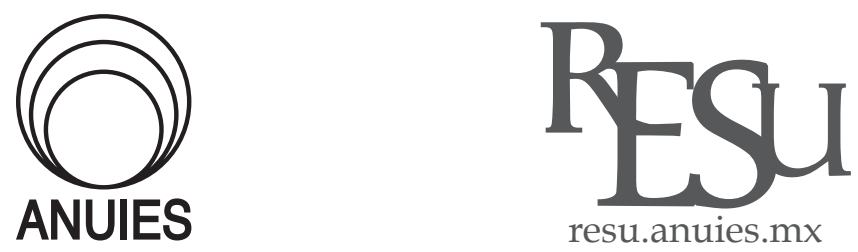

REVITA DEU
EDLCACIÓN
SUPERIOR

ARTí́CULO

\title{
El sector de la investigación en México: entre privilegios, tensiones y jerarquías*
}

\section{The Mexican research sector: privileges, tensions and hierarchies}

\author{
Marion Lloyd** \\ * Agradezco a mis colegas en el Seminario de Educación Superior de la UNAM, Jesús Galaz Fontes, Manuel Gil \\ Antón y Jorge Martínez Stack, por proporcionarme el acceso a la base RPAM-SNI de forma inédita. \\ ** Dirección General de Evaluación Institucional, Universidad Nacional Autónoma de México. Ciudad de \\ México, México. Correo electrónico: marionlloyd@gmail.com
}

Recibido el 28 de julio del 2017; aceptado el 20 de marzo del 2018.

\begin{abstract}
Resumen
Este artículo analiza los impactos de las políticas de ciencia y tecnología en México de las últimas décadas sobre el sector de la investigación. Para ello, se presenta una selección de datos sobre los investigadores provenientes de las dos encuestas más extensas acerca del tema. De particular relevancia, se divulgan por primera vez los resultados de la segunda encuesta, aplicada por la Red de Investigadores sobre Académicos (RDISA) en 2007-2008 a casi 12 mil miembros del Sistema Nacional de Investigadores (SNI). A pesar de haberse realizado hace una década, es el estudio más completo y reciente sobre los miembros del SNI. A su vez, argumento que probablemente refleja el estado actual de los investigadores, debido a la continuidad de las políticas en años recientes. Da una imagen compleja, revelando fuertes tensiones, jerarquías y desigualdades de género, así como el grado de privilegio que gozan los miembros del sNi comparado con los demás académicos del país.
\end{abstract}

Palabras clave: Políticas de ciencia y tecnología; Sistema Nacional de Investigadores; Sociedad de conocimiento; Investigadores mexicanos; Jerarquías académicas 


\begin{abstract}
This article analyzes the impact of several decades of science and technology policies in Mexico on the country's research sector, using a selection of data from the two most extensive surveys of Mexican researchers. Of particular relevance, I present for the first time the results of the second survey, applied by the Network of Researchers on Academics (RDISA) in 2007-2008 to nearly 12,000 members of the National Researchers System (SNI). Although the study was conducted a decade ago, it constitutes the most complete and recent survey of the SNI members. Given the continuity in S\&T policies in recent years, the data likely reflects, in broad terms, the current state of Mexican researchers. It gives a mixed picture, revealing strong tensions, hierarchies and gender inequalities, as well as the significant privileges enjoyed by SNI members compared with other Mexican academics.
\end{abstract}

Keywords: Science and technology policies; Sistema Nacional de Investigadores; Knowledge society; Mexican researchers; Academic hierarchies

\title{
Introducción
}

\begin{abstract}
A nte las demandas de la llamada "sociedad de conocimiento", el sector de la investigación ha adquirido una nueva relevancia en la mayoría de los países, incluyendo México (Marginson y Ordorika, 2010). A pesar del debate en torno a la adecuación del término para las economías emergentes - que enfrentan fuertes desventajas en cuanto al acceso a conocimientos y tecnologías (Carreón y Melgoza, 2012) - , existe un consenso en torno a lo que define esta nueva fase de desarrollo económico a nivel mundial:
\end{abstract}

Una de las características de la sociedad contemporánea es el papel central del conocimiento en los procesos de producción, en la medida en que el término calificativo más utilizado es el de la sociedad de conocimiento. Estamos presenciando la emergencia de un nuevo paradigma económico y productivo en que el factor más importante deja de ser la disponibilidad de capital, mano de obra, materia prima o energía, y se convierte en el uso extensivo del conocimiento y de la información. Hoy, las economías más avanzadas se basan en la mayor disponibilidad de conocimiento. Cada vez más, las ventajas comparativas son determinadas por el uso competitivo del conocimiento y de las innovaciones tecnológicas (Bernheim y Chaui, 2003:1).

Ante este panorama, durante las últimas décadas, el gobierno mexicano ha diseñado políticas públicas que buscan fortalecer el ámbito científico en el 
país. Destaca la implementación desde la década de 1980 de los llamados programas de "pago por mérito", que buscan promover la productividad de los académicos a través de estímulos económicos; el primer programa fue dirigido a los investigadores y después se crearon sistemas para fomentar la calidad de la docencia en las universidades públicas (Izquierdo, 1998).

El pionero en México de estos programas fue el Sistema Nacional de Investigadores (SNI), que fue creado en 1984 como medida de contención ante el desplome de hasta $60 \%$ en los salarios de los académicos durante la llamada “década perdida" a partir de 1980 (Galaz y Gil Antón, 2009; Ruíz, 2014), y el cual es administrado por el Consejo Nacional de Ciencia y Tecnología (Conacyt), institución fundada en 1971 para formular y administrar la política científica en el país. A más de tres décadas de su implementación, existen numerosos análisis respecto al impacto del SNI sobre el sector de la investigación en México; sin embargo, hay una escasez de información a nivel nacional que permitiría una caracterización más completa del entorno y de sus integrantes.

En este artículo presento por primera vez los resultados de una encuesta que fue enviada en 2007-2008 a más de 14000 miembros del SNI, obteniendo más de 5000 respuestas; a pesar de ser el estudio más completo sobre los miembros del sistema, por distintos motivos sus hallazgos nunca han sido diseminados. ${ }^{1}$ La encuesta permite rellenar algunas de las grandes lagunas que existen acerca del ámbito de la investigación en México, al proveer cientos de datos relacionados con las trayectorias y percepciones de sus integrantes.

Antes de entrar en los detalles de la encuesta, es necesario poner en contexto las políticas de fomento a la ciencia y la tecnología en México aplicadas durante las últimas cuatro décadas, entre las cuales el SNI aparece como una parte central, y sobresalen las siguientes: los proyectos de repatriación de investigadores durante la crisis económica en la década de 1980; las becas para el posgrado (tanto nacional e internacional) que aumentaron significativamente a partir de la década de 1990; la creación en 1991 de un esquema de evaluación a los posgrados a través del Padrón de Posgrados de Excelencia (después Programa Nacional de Posgrados de Calidad); las becas para fomentar la profesionalización de los profesores; y las nuevas plazas para “jóvenes investigadores" a partir de 2014, entre otras (Olivares, 2014; Covarrubias, 2017).

Tales políticas se insertan en la lógica de la Nueva Gestión Pública (New Public Management, o NPR por sus siglas en inglés), una tendencia en la administración pública que emergió primero en los países anglosajones en los

${ }^{1}$ Agradezco a mis colegas del Seminario de Educación Superior de la UNAM, Jesús Galaz Fontes, Manuel. 
años 80, para después extenderse a gran parte del mundo; se caracteriza por un nuevo énfasis en la rendición de cuentas, en los esquemas de evaluación - el llamado "Estado evaluador" - y en la racionalización (y recorte) del gasto público. El enfoque va de la mano con las políticas neoliberales impulsadas por Estados Unidos y los organismos internacionales, como el Banco Mundial y el Fondo Monetario Internacional, también a partir de la década de 1980 (Field, 2015).

Un ejemplo claro de la lógica del New Public Management son los programas de pago por mérito, también llamados de estímulos o de pago por desempeño. Aunque estos mecanismos no son nuevos a nivel mundial - los primeros sistemas fueron implementados en Estados Unidos a principios del siglo xx para los maestros de la educación básica y después se extendieron a la educación superior - , su adopción en América Latina data apenas de las décadas de 1980 y 1990 (Izquierdo, 1998); operan bajo la premisa de que a mayor incentivo económico, mayor productividad y mejor calidad del servicio (Field, 2015). En el caso de la educación superior, los programas buscaban crear un nuevo actor académico, infundido con los valores de la competencia internacional (Gérard y Grediaga, 2009):

Cada uno de los componentes del pago sobre desempeño - incluyendo las representaciones cuantitativas del "desempeño"; el control sobre atributos como "calidad", los cuales son medidos; la presión sobre los académicos para entregar productos que están alineados con las prioridades estratégicas de las universidades; el impulso a los académicos para entrar en competencias anuales de suma cero; y la desacreditación de cualidades humanas como el compromiso y la creatividad - encaja cómodamente con la ideología de NPM (Field, 2015: 5).

\section{El debate sobre los programas de pago por mérito}

A pesar de la hegemonía de la nueva cultura de evaluación a nivel internacional, en años recientes han surgido fuertes debates en muchos países sobre los mecanismos de pago por mérito; una parte de estas críticas cuestiona la eficacia en lograr su cometido principal: mejorar la productividad y la calidad en la educación superior. A su vez, se han enfatizado los efectos indeseados (o perversos) de las políticas, tanto en términos personales como institucionales (Christensen y Manley, 2011). No obstante, mientras existen muchos estudios sobre el impacto de los programas en la educación básica, hay una escasez de información sobre el sector de la educación superior, en 
donde tales mecanismos son más recientes. Gran parte de estos estudios han sido realizados en Estados Unidos, país que adoptó estos programas por primera vez en el nivel superior a partir de la década de 1970 (Izquierdo, 1998).

McCrea y Deyrup (2016), en un estudio realizado dentro de una universidad pública de investigación en Estados Unidos, encontraron que los programas tuvieron un mayor impacto sobre el nivel de investigación de los profesores que en la calidad de su docencia; también percibieron diferencias de género, con una mayor aceptación entre los hombres que entre las mujeres; $y$, por otro lado, hallaron los siguientes efectos negativos de las políticas: “una reducción en el nivel de colegialidad, un incremento en acciones políticas u otros comportamientos disfuncionales, problemas de evaluación, sesgos institucionales, y el costo de la implementación" (p. 4); finalmente, concluyeron que el impacto de los programas depende mucho de la forma como están implementados, de la claridad de los criterios de evaluación, del diseño y del grado de participación de los propios académicos: "Para una compensación basada en la productividad, el diablo realmente está en los detalles" (McCrea y Deyrup, 2016: 1).

Otro estudio realizado por Christensen y Manley (2011), también en Estados Unidos, dedujo que existe poca relación entre los sueldos percibidos por los académicos inscritos en estos programas y su nivel de productividad científica. Mientras tanto, Terpstra y Honoree (2009) apreciaron un "efecto algo positivo" sobre el desempeño de los académicos en las áreas de docencia, de investigación y de extensión universitaria, después de aplicar una encuesta a casi 500 académicos en universidades alrededor del país; sin embargo, aclararon que existe mayor información sobre las percepciones negativas de los académicos sobre los programas que respecto a la eficacia de los mismos.

Estudios en Europa han encontrado resultados parecidos: una leve mejoría en el nivel de productividad, en términos cuantitativos, así como numerosos efectos perversos (véase, por ejemplo, Minasyan, et al., 2017). Entre los efectos indeseados de las políticas, citados por muchos autores, están: la simulación en el cumplimiento de requisitos de productividad; la reducción de la colegialidad de la academia; un aumento en el estrés sufrido por los académicos debido a la cultura de "publicar o morir"; la institucionalización de sesgos de género, de raza, o de campo disciplinario; y la creciente estratificación y polarización de los académicos, entre la mayoría que se dedica a la docencia y una minoría privilegiada abocada a la investigación (McCrea y Deyrup, 2016; Christensen y Manley, 2011). 


\section{El caso mexicano}

Los estudios realizados en México también han identificado efectos parecidos emanados de las políticas (véase García, 2001; Galaz y Gil, 2009; Gérard y Grediaga, 2009; Galaz y Gil, 2013), entre éstos resalta la creciente polarización de la academia, ya que, en general, las políticas gubernamentales en México han premiado en mayor medida a los investigadores que a los docentes (Ordorika y Navarro, 2006).

La creación del SNI es un claro ejemplo del trato preferencial hacia los académicos. Aunque el programa fue concebido como una medida temporal, para frenar la "fuga de cerebros" durante la crisis económica en la década de 1980, después se institucionalizó como pieza angular de la política más relevante para el sector de la investigación (Galaz y Gil, 2009). El sistema comenzó con 1396 miembros, mientras que para 2017 hubo unos 25000 investigadores inscritos (Rodríguez, 2017) distribuidos en cinco niveles (candidato, nivel I, II, III, y emérito). Con el tiempo, el programa también incorporó a extranjeros radicados en México y mexicanos trabajando en universidades fuera del país.

Como su nombre sugiere, el programa está dirigido a los académicos cuya labor principal es la investigación científica. El sNi otorga becas mensuales a investigadores con un alto nivel de producción científica, estímulos que pueden hasta duplicar el sueldo de los integrantes. Los miembros son sometidos a evaluaciones cada tres años para determinar si permanecen o suben de nivel. Deben constatar el cumplimiento con las cuotas de productividad, definidas por el número de publicaciones en revistas indexadas o de libros en editoriales de reconocido prestigio, la producción de patentes, la formación de recursos humanos, entre otras áreas.

Como veremos más adelante, el sistema mexicano resulta notable por el peso preponderante que representan los procedimientos de pago por mérito en los sueldos de los académicos, y de los investigadores en particular (Galaz y Gil Antón, 2013). Tal diseño tiene implicaciones para la seguridad económica y laboral de los miembros, ya que sus sueldos dependen en gran medida de las reglas del sistema y de las evaluaciones de sus pares. A su vez, los pagos son clasificados como "becas" y no como sueldos, y pueden ser suprimidos en cualquier momento; de igual forma, la mayoría de los participantes - con excepción de los pocos que logran el estatus de emérito - no gozan de la beca a partir de su jubilación. Como resultado, el sistema otorga beneficios a corto o mediano plazo, pero no garantiza la estabilidad económica de sus integrantes. 
En contraste, en Brasil, la mayoría de los sueldos de los académicos proviene directamente de su institución, y los jubilados siguen percibiendo la mayor parte de su sueldo al retirarse de su universidad. Inclusive los académicos brasileños pueden solicitar becas al gobierno federal para realizar investigación, pero deben destinar la mitad de los fondos a la realización del proyecto (Lloyd, 2013).

Sin embargo, los programas de pago por mérito forman sólo una parte de la estrategia mexicana en materia de ciencia y tecnología. Por otro lado y también de gran importancia fue la creación, en 1996, del Programa de Mejoramiento del Profesorado (Promep) (ahora Prodep), un programa que otorga becas para los estudios de posgrado dirigido a los profesores mexicanos de tiempo completo, y que promueve la formación de cuerpos académicos y redes de colaboración entre investigadores, tanto a nivel nacional como internacional (Gobierno de México, 2017). De igual manera, el gobierno ha creado nuevos centros de investigación en distintas partes del país, con el fin de descentralizar y adecuar la producción de conocimientos según las necesidades locales y globales; e incluso se ha incrementado de forma sustancial el apoyo a los programas de posgrado a través del otorgamiento de becas a estudiantes de maestría y de doctorado, las cuales se duplicaron, entre 2006 y 2017, de 34000 a más de 72000 (Conacyt, 2018).

En el proceso, el gobierno mexicano ha promovido la investigación como la labor principal de una parte de los académicos (Galaz y Gil, 2009), sobre todo en las universidades y los centros públicos de investigación (EXECUM, 2017). En general, los investigadores - y, sobre todo, los miembros del SNIrepresentan una nueva clase privilegiada dentro de la academia mexicana, tanto en términos de sus ingresos económicos como en su nivel de prestigio (Didou y Gérard, 2010, 2011; Galaz, et al., 2012; Balbachevsky, 2015). No obstante, los privilegios van acompañados de nuevos criterios y responsabilidades:

El sNi reestructuró las élites científicas, imponiéndoles dos exigencias vigentes hasta la fecha: la de la obtención de un doctorado a edades cada vez más tempranas y la de la adquisición del máximo grado escolar en polos de excelencia, internacionales primero, nacionales ahora (Didou y Gérard, 2011: 32).

Aunado a estos requisitos, aparecen las nuevas presiones por producir conocimiento, el cual es medido a través de la publicación de textos en revistas y en libros arbitrados que posean reconocimiento a nivel internacional. Tal lógica de "publicar o morir", que subyace en los esquemas de pago por mérito, ha tenido fuertes repercusiones entre la academia mexicana, y en parti- 
cular para el sector de la investigación (Galaz y Gil, 2009; Gérard y Grediaga, 2009). Esta particularidad influye en las decisiones y trayectorias laborales de los investigadores, quienes enfrentan fuertes presiones por competir, muchas veces en condiciones desiguales. Los criterios del sistema favorecen a los investigadores de instituciones consolidadas y a los miembros de las llamadas "ciencias duras", ya que existen fuertes "sesgos disciplinarios" en los mecanismos de evaluación (Didou y Gérard, 2010: 64); por ejemplo, una mayoría de las revistas indexadas a nivel internacional pertenecen a las ciencias duras (Ordorika y Lloyd, 2014), de modo que los investigadores de estos campos también son más propensos a estudiar fuera, particularmente en los países anglosajones, mientras que los de las ciencias sociales y humanidades suelen escoger los países del continente europeo (Didou y Gerard, 2011). A su vez, como veremos más adelante, hay fuertes sesgos de género que impactan en las posibilidades que tienen las mujeres de subir los peldaños del sistema.

En este artículo, analizo las trayectorias y percepciones de los investigadores en México, con base en las dos encuestas más extensas y recientes sobre el sector; busco dar una breve caracterización de los miembros del sistema en los años 2007-2008, con el fin de evaluar el impacto del programa en la configuración del subsector de la investigación en el país; presto particular atención a temas como la división de género, el nivel de educación alcanzado, el país en donde fue obtenido el grado, los sueldos percibidos, el grado de movilidad internacional, así como las percepciones sobre su labor de investigación.

La primera encuesta fue aplicada por la Red de Investigadores sobre Académicos (RDISA) en 2007-2008 a casi 3000 académicos de tiempo completo en México. Formó parte de la encuesta internacional The Changing Academic Profession (CAP), destinada a los académicos de 18 países y un territorio (Hong Kong). Actualmente, en 2018, se está desarrollando una segunda edición del CAP a nivel internacional, sin embargo, aún no hay una fecha para la divulgación de los resultados. Los datos de la primera encuesta ya han sido difundidos ampliamente, permiten hacer comparaciones internacionales $\mathrm{y}$, de particular importancia, sirven como referencia para analizar los hallazgos de una segunda encuesta, mucho más amplia, que se enfocó en los miembros del SNI.

La segunda encuesta fue enviada por la RDISA durante los mismos años a los 14681 miembros del SNI que hubo en ese entonces (Conacyt, 2015a). Al final se obtuvieron respuestas (válidas) de 5426 investigadores, en lo que representa el estudio más extenso (y reciente) sobre el SNI. Los resultados arrojan una luz valiosa sobre el ámbito de la investigación en México, dejando ver tanto los aciertos como los efectos inesperados (o perversos) de las políticas. Los resultados de las encuestas tienen implicaciones para la academia 
en su conjunto por los esfuerzos gubernamentales para crear una economía de conocimiento en el país.

La primera sección del presente texto da un panorama general del estado de la investigación en el país en la segunda década del siglo XXI. Después, reviso algunos de los estudios recientes sobre trayectorias laborales de los investigadores. En la tercera parte, el núcleo de este artículo, analizo una selección de datos provenientes de las dos encuestas realizadas por la RDISA, prestando particular atención a la segunda encuesta y al subgrupo conformado por los miembros del SNI. A continuación, presento algunos datos más recientes sobre el SNi y el sistema de ciencia y tecnología (CyT) en el país, como punto de referencia para analizar la evolución y la continuidad del sistema en los últimos años. Finalmente, resumo algunos de los hallazgos más relevantes sobre el sector de la investigación en México, a más de dos décadas de la creación del primer programa de pago por mérito en el país.

\section{El panorama actual del sistema de la investigación en México}

No obstante la cantidad de políticas orientadas a fomentar la ciencia y la tecnología en México, el sector de la investigación es aún un espacio en proceso de configuración (Galaz y Gil, 2009) y de consolidación (Didou y Gérard, 2011). Todavía no se puede hablar de una masa crítica de investigadores, como la que existe en Estados Unidos o en los países europeos. En México hay 0.84 investigadores por cada cien mil personas económicamente activas, contra nueve en España, según los datos más recientes de 2014 (RICYT, 2017). La cifra es aún baja cuando se compara con otros países latinoamericanos: en 2014, Argentina reportó 4.76 investigadores por cada cien mil PEA, Brasil 2.3 (cifra de 2010), y Chile, 1.16 (RICYT, 2017).

Tal situación se debe en gran medida a la poca inversión en CyT en México, tanto por parte del gobierno como de la esfera privada. La escasez de fondos para la investigación es particularmente sorprendente, debido a que, desde 2003, una serie de leyes ${ }^{2}$ y programas gubernamentales mandatan un mínimo global del 1\% del PIв para el sector. En 2015, México invirtió 0.53\% en investigación en CyT, contra 1.28\% en Brasil, país que es el indiscutible líder regional, tanto en el nivel de su inversión como en su producción científica (Lloyd, 2017; RICYT, 2018).

2 Tales disposiciones incluyen La Ley de Ciencia y Tecnología de 2003 y los distintos Programas Especiales de CyT. 
Como hicieron sus antecesores, el presidente Enrique Peña Nieto (20122018) se comprometió a dar un impulso inédito al sector de la ciencia y la tecnología, es decir, a convertir a México en una sociedad de conocimiento en todos los sentidos. El Programa Especial de Ciencia, Tecnología e Innovación 2014-2018 (PECITI), del Gobierno Federal, declara lo siguiente: “Existe la convicción de que la inversión en ciencia y tecnología es una herramienta fundamental para acceder a una economía de bienestar, basada en el conocimiento" (Conacyt, 2014). El plan fija metas generales y específicas en la materia, las cuales incluyen incrementar el gasto público para llegar a una inversión del 1\% del PІв en CyT, aumentar el número de científicos miembros del Sistema Nacional de Investigadores en las áreas STEM (ciencia, tecnología, ingeniería y matemáticas), fomentar la incorporación de jóvenes doctores en las universidades y centros de investigación públicos, y facilitar la movilidad (nacional e internacional) de estudiantes de posgrado y de investigadores.

El programa también ofrece un panorama del estado del entorno académico en México en 2012, que sirvió como base para la formulación de las metas sexenales. En ese año, los investigadores mexicanos publicaron 10181 artículos en revistas indexadas por el Thomson Reuters Web of Science (ISI), lo que representó menos de la tercera parte de los artículos publicados por investigadores brasileños, que fue de 35 042. La brecha con los países desarrollados fue aún mayor: en Estados Unidos la cifra ascendió a 255072 artículos, y a 96692 en el Reino Unido (Conacyt, 2014).

El PECITI busca atender una serie de problemáticas que enfrenta el sector de la investigación en México, lo cual comprende la falta de apoyo económico y de oportunidades laborales, el bajo nivel de producción de artículos y de patentes, la poca colaboración internacional, entre otros temas. No son problemas nuevos. Desde los años 90, muchos especialistas han señalado las deficiencias en las políticas de fomento a la ciencia y la tecnología en México (García, 2000, 2001; Canales, 2007, Didou y Gérard, 2010). Entre los problemas señalados asoman la sobre-burocratización de los procesos de evaluación (el Estado evaluador), la cual estorba la producción científica y propicia la "simulación" por parte de los académicos (García, 2001); la falta de políticas de equidad de género; la alta concentración de investigadores en algunos estados e instituciones, pues en 2016, 70\% de los miembros del sNI se concentraba en 60 IES y centros de investigación, y 33\% laboraba en cinco de ellos ubicados en la Ciudad de México y Jalisco (ExeCum, 2018). 


\section{Trayectorias laborales y movilidad de los investigadores}

Gran parte de estos análisis se basan en los estudios de las trayectorias laborales de los investigadores (Galaz y Gil, 2009; Gérard y Grediaga, 2009; Didou y Gérard, 2010; Galaz, De la Cruz, y Rodríguez, 2010; Chavoya, 2013). Éstos permiten ver factores como los niveles de ingresos y de satisfacción laboral, así como el grado de movilidad, tanto vertical (ascensión laboral) como horizontal (entre instituciones en México o el extranjero) de los académicos, según las características personales de los mismos.

El tema de la movilidad resulta de suma importancia para las políticas de ciencia y tecnología. En las últimas décadas, el gobierno mexicano ha enviado a docenas de miles de académicos mexicanos a formarse en distintas universidades del país y en el extranjero, con el fin de aumentar su capacidad de realizar investigación de punta. No obstante, un número desconocido de esos becarios no regresa. El Consejo Nacional de Ciencia y Tecnología (Conacyt), que está a cargo de administrar el SNI y de otorgar becas a nivel posgrado, estima que hay $5 \%$ de "fuga de cerebros" entre becarios, la mitad de maestría y la mitad del doctorado. Según tales estimados, Estados Unidos es el mayor país receptor de mexicanos con altos niveles de estudios, $60 \%$ del total, seguido por los países europeos con 26\%, y Canadá con 5\% (Marmolejo, 2009).

Marmolejo, quien actualmente coordina el área de educación superior del Banco Mundial, señala la falta de información confiable sobre el número de académicos mexicanos radicados en el extranjero. Por ejemplo, el American Community Survey estimó que para 2005 había 75000 mexicanos con estudios de posgrado en Estados Unidos (Marmolejo, 2009). Otro estudio de Escobar y Martin (2006) postuló que 19\% de los varones mexicanos con maestría y 29\% de las mujeres con ese nivel, vivían en Estados Unidos; entre los que contaban con doctorado, la proporción fue de 32 y 39\%, respectivamente (citado en Marmolejo, 2009), e incluso: “De ser generalizables estos resultados, se podría inferir que hay un colateral y paradójico efecto asociado con las políticas de otorgamiento de becas para estudios de posgrado en el extranjero" (Marmolejo, 2009: 107).

Actualmente, el tamaño real de la "fuga de cerebros" se ha vuelto un tema de considerable debate. Algunos especialistas (Didou y Gérard, 2009; Tuirán y Ávila, 2013) argumentan que más bien se debería hablar de una "circulación de cerebros", ya que la movilidad puede ocurrir en distintas direcciones o no ser permanente. El nivel de movilidad tampoco es homogéneo, sino que varía según las disciplinas (Didou y Gérard, 2011). 


\section{Las encuestas sobre investigadores en México}

Debido a la escasez de información oficial sobre los investigadores mexicanos, son particularmente útiles las dos encuestas nacionales aplicadas entre 2007 y 2008 por la RDISA, en el marco del proyecto La Reconfiguración de la Profesión Académica en México (RPAM), la contribución mexicana al proyecto internacional The Changing Academic Profession. Las encuestas, en las que participaron distintas esferas de la academia mexicana, representan la fuente de información más reciente y extensa sobre las características personales y el entorno laboral de los investigadores en el país. A su vez, permiten dar cuenta de algunos cambios en el sector a través de las políticas públicas de CyT adoptadas en las últimas décadas.

La primera encuesta se envió a 2826 académicos, que respondieron 1775 de ellos (90\% de tiempo completo) en 81 Instituciones de Educación Superior (IES) en México (Galaz, De la Cruz, y Rodríguez, 2010). El cuestionario incluyó cientos de preguntas sobre las características personales y profesionales de los investigadores, su formación académica, sus trayectorias laborales y sus percepciones sobre sus lugares de trabajo y de profesión. La primera encuesta RPAM ha dado como resultado una extensa producción ${ }^{3}$ en revistas y libros a nivel internacional sobre la conformación de la profesión académica en México y otros países; sirve como punto de referencia para contrastar a los académicos en general con los miembros del SNI; también resulta de particular relevancia para este análisis, pues permite examinar los resultados de la segunda encuesta en el contexto de la academia mexicana en general.

La segunda encuesta se enfocó específicamente en el sector de la investigación en México y abarcó una población varias veces mayor. Se envió el mismo cuestionario del RPAM a los 14576 académicos que formaban parte del SNI en 2007-2008, y se obtuvieron respuestas de 5426 investigadores. ${ }^{4}$ A pesar de la gran riqueza de información disponible en la encuesta, por distintos motivos, hasta el momento no se han diseminado los resultados. Por ello, los datos contenidos allí sirven como una fuente privilegiada de información sobre el entorno de la investigación en México.

\footnotetext{
${ }^{3}$ En México, véase los trabajos de Galaz, Gil, Padilla, Estévez, Martínez, y Lloyd, entre otros. A nivel internacional, destaca la serie de libros editados por Cummings, Teichler, y otros, bajo el título de The Changing Academy-The Changing Academic Profession.

${ }^{4}$ Jesús Galaz, comunicación personal, el 30 de marzo, 2017.
} 


\section{Sobre la metodología}

En el diseño de la encuesta RPAM-CAP se tradujeron y se modificaron las preguntas para adecuarlas al contexto del país. Se realizó la muestra con base en los datos del Formato 911 de la Secretaría de Educación Pública, la fuente oficial de información estadística sobre la educación superior en México. De un total de 2029 instituciones y 255274 académicos a nivel nacional, finalmente se redujo el universo de estudio a 379 instituciones de educación superior que cumplieron los siguientes criterios: que tuvieran más de 20 académicos de tiempo completo o medio tiempo y que ofrecían títulos de cuatro años (en vez de dos). De esta forma, se fijó un universo total de 79389 académicos de TC/MT, de los cuales se obtuvieron los 1775 respuestas finales (Galaz, et al., 2009). Cabe señalar que, por el diseño de la muestra, quedaron excluidas las instituciones tecnológicas y las IES privadas menos prestigiadas, las llamadas "de absorción de demanda residual" (Gil Antón, 2008). No obstante, tal hecho no afecta al análisis del subgrupo del SNI, ya que esas instituciones no suelen contar con muchos miembros del sistema.

De acuerdo con los fines de este artículo, se adoptaron varias estrategias metodológicas para el análisis de la primera base. Existe dificultad en determinar quiénes pertenecen al sistema de investigación en los distintos países, ya que la mayoría de los académicos de tiempo completo reporta realizar investigación como una de sus labores académicas. Por ello, realicé distintos recortes para acercar los diferentes grupos de investigadores, empleando el software estadístico sPss. Por ejemplo, utilicé la membresía en el SNI como proxy para los investigadores al realizar la comparación con los demás académicos mexicanos. Al hacer una breve comparación entre los investigadores mexicanos y los de otros países, en donde no existe el equivalente al sNI, separé a los investigadores que realizan por lo menos 20 horas de investigación por semana (la cantidad promedio que reportan los miembros del SNI).

Juntas, las dos encuestas levantadas por parte de la RDISA permiten tener un panorama bastante amplio sobre la condición laboral de los investigadores en el país en los años 2007 y 2008. A pesar de que ha pasado una década desde su realización, argumentaría que es posible hacer inferencias para el periodo actual, debido a la continuidad de la mayoría de las políticas gubernamentales durante los últimos sexenios. En la sección final, resumo algunos de los datos disponibles para el periodo actual, que sirven como punto de referencia para ver la evolución del sistema en los últimos años. 


\section{La Encuesta RPAM-CAP}

La encuesta Changing Academic Profession, que mide más de 400 variables, permite realizar comparaciones muy variadas entre los académicos de distintos países o entre subgrupos dentro de un mismo país. En este caso, comparo a los miembros mexicanos del SNI ( $\mathrm{n}=367)$ y los no miembros $(\mathrm{n}=1448)$, para poder hacer una caracterización inicial del sector de la investigación en México.

\section{Perfil general de los encuestados}

Entre los 367 miembros del SNI incluidos en la base, la gran mayoría (68\%) son hombres. Es un dato importante, ya que, como veremos más adelante, refleja un sesgo de género en el ámbito de la investigación en México (Didou y Gérard, 2011). En comparación, la preponderancia de hombres entre los académicos no miembros es un poco menor: $62 \%$.

Los investigadores también se distinguen por ser un grupo más internacional: $15 \%$ nació en el extranjero, comparado con menos de $2 \%$ del otro sector. A su vez, 43\% del primer grupo reportó haber vivido en distintos países desde que obtuvo su primer grado académico, contra $17 \%$ del segundo. Es probable que la movilidad de la mayoría de los miembros SNI ocurrió durante su periodo de estudios de posgrado. Entre los investigadores, 93\% reportó contar con el doctorado y $21 \%$ con el posdoctorado, contra 16 y $0.7 \%$ del grupo docente, respectivamente. Entre los miembros del sNI con doctorado, $46 \%$ lo estudió en el extranjero; predominó Estados Unidos con 15\% de los doctorantes, seguido por Francia (7.5\%), España (7.4\%), y Reino Unido (5.8\%), y el resto fue distribuido por otros 14 países. Finalmente, entre los miembros del SNI que realizaron un posdoctorado ( $\mathrm{N}=78)$, sólo $13 \%$ lo hizo en México, en cambio, $41 \%$ estudió en Estados Unidos, seguido por Canadá (9.2\%), Francia (9\%) y Reino Unido (7.8\%).

El mayor nivel de experiencia internacional entre los miembros del SNI es relevante, ya que señala el nivel de privilegios que han gozado este grupo comparado con sus pares no miembros. También indica que el sistema premia cierto tipo de perfil entre sus integrantes, otorgando mayor prestigio a los títulos obtenidos en el extranjero (Gérard y Grediaga, 2009). 
Figura 1

Países receptores de estudiantes de doctorado

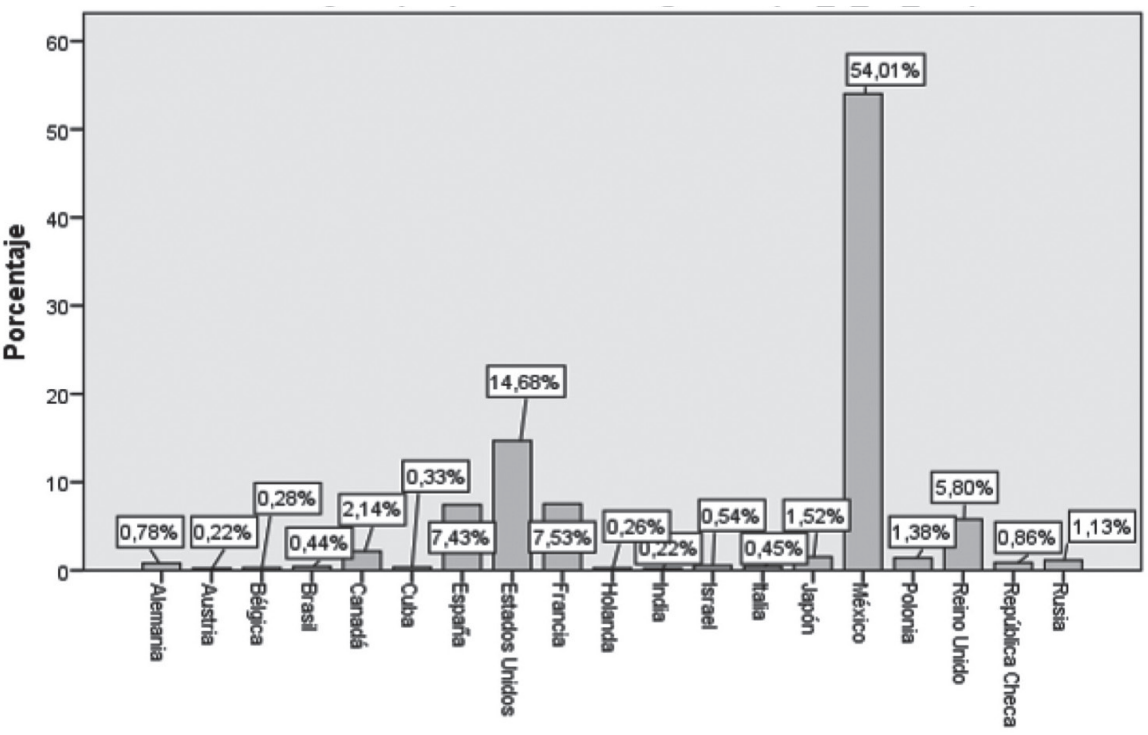

Doctorado: ¿En qué país obtuvo el grado? (A1_8_4a_rcN)

Casos ponderados por Ponderador a la muestra usable

Fuentes: Base RPAM-CAP 2007-2008.

En cuanto a las condiciones laborales, resalta el hecho de que los miembros del sNi ganaron en promedio casi el doble de lo que percibieron los académicos que no fueron miembros del sistema: USD $\$ 48700$ al año contra USD $\$ 25$ 160. Esta diferencia obedece mayormente a los estímulos que recibió el primer grupo a través del sNI y, en menor grado, de los programas institucionales; los investigadores reportaron recibir un promedio anual de USD $\$ 12800$ y USD $\$ 11400$, respectivamente, por estos conceptos. Cabe señalar que a pesar de las reformas recientes que buscan dar mayor énfasis a la labor docente, los estímulos institucionales, al igual que los del SNI, siguen privilegiando la labor de investigación (Buendía et al., 2017). Tal discrepancia tiene razones prácticas, además de ideológicas:

Es más fácil contar las publicaciones que evaluar los resultados de las labores docentes, por lo que éstas se han menospreciado entre los indicadores, minimizando el esfuerzo que los académicos destinan a ellas [...]. Esta condición genera, a su vez, que los programas sobrevaloren ciertas actividades sobre otras - por ejemplo, la investigación por encima de la docencia, la difusión de la cultura y la vinculación social - sin analizar los aportes y su calidad en el marco de los campos de conocimiento o institu- 
cionales, el tipo de resultados, las tradiciones disciplinarias y la etapa de la trayectoria de los sujetos evaluados (Buendía et al., 2017, s.p.).

Tal hecho tiene implicaciones para la calidad de la docencia en México. En la práctica, promueve que los académicos con mayor nivel de estudios (y sobre todo los que fueron educados en el extranjero) opten por dedicarse a la investigación, o busquen acomodo en los centros de investigación especializados, o adquieran la figura de investigadores de la Universidad Nacional Autónoma de México. En contraste, en Brasil, aun en las universidades de investigación de mayor nivel como son la Universidad de São Paulo o la Universidad Estatal de Campinas, la mayoría de los académicos tiene la figura de profesor-investigador, una política gubernamental que busca incorporar la investigación dentro del aula (Lloyd, 2013).

Los miembros del SNI reportaron invertir 20.3 horas a la semana en investigación, comparado con sólo siete horas por parte del grupo docente; tal cifra no es casual, ya que el SNI estipula un mínimo de 20 horas semanales dedicadas a la investigación. No obstante, los requisitos que impone el programa tienen su costo en términos de un mayor estrés laboral. Al responder a la afirmación, "Mi trabajo es fuente de una considerable tensión personal", $29 \%$ de los miembros del SNI estuvo muy de acuerdo o de acuerdo, comparado con $21.5 \%$ de los no miembros.

Otro punto interesante de observar es el nivel de capital cultural de ambos grupos, en el sentido del entorno educativo y cultural en que fueron criados (Bourdieu, 1986); en este caso, utilicé el nivel educativo del padre como proxy para el capital cultural de los encuestados. Entre el grupo SNI, 23\% reportó que su padre había estudiado hasta el nivel licenciatura y $8 \%$ hasta el posgrado (parcial o completo); entre el otro grupo, las cifras fueron 19 y 6\%, respectivamente. Es decir, en general, los miembros del SNI vienen de familias con mayor nivel educativo, otro ejemplo de su estatus privilegiado.

Tabla 1

Comparativo miembros SNI/ No SNI

\begin{tabular}{lll}
\hline & Miembros sNI & No sNI \\
\hline Hombres & $68 \%$ & $62 \%$ \\
\hline Extranjeros de nacimiento & $15 \%$ & $2 \%$ \\
\hline Doctorado & $93 \%$ & $21 \%$ \\
\hline Posdoctorado & $16 \%$ & $0.7 \%$ \\
\hline Sueldo anual (UsD) & $\$ 48700$ & $\$ 26160$ \\
\hline Promedio de horas/semana en investigación & 20.3 & 7 \\
\hline
\end{tabular}

Fuente: elaboración propia, con datos de la base RPAM-CAP 2007-2008. 


\section{Comparación con investigadores de otros países}

La encuesta también permite realizar comparaciones con investigadores de otros países. Son particularmente relevantes los casos de Brasil y de Argentina, los otros dos países latinoamericanos que participaron en el proyecto CAP; también son los principales rivales regionales de México en materia científica (Martínez, Lloyd, y Ordorika, 2015). Entre el subgrupo que reportó realizar por lo menos 20 horas de investigación a la semana, los investigadores mexicanos tuvieron una representación mucho menor de mujeres: 33\%, comparado con $44 \%$ en Brasil y 56\% en Argentina. Sin embargo, cabe señalar que la preponderancia de hombres entre los investigadores en México, de $67 \%$, fue casi igual que en Estados Unidos, donde éstos representaban $68 \%$ del subgrupo. En cuanto al nivel de estudios de los investigadores, México se ubicó en el promedio entre Brasil y Argentina; 80\% de los brasileños obtuvo el doctorado y $30 \%$ el posdoctorado, mientras que en México las cifras fueron de 70 y 17\%, y en Argentina, 49 y 15\%, respectivamente.

Por otro lado, los mexicanos registraron el mayor nivel de movilidad internacional durante sus estudios de doctorado y posdoctorado. Entre los mexicanos con doctorado, $42 \%$ estudió ese nivel en el extranjero, comparado con $19 \%$ en Brasil y $16 \%$ en Argentina. Es probable que la mayoría de los mexicanos de ese grupo fueran beneficiarios de las más de 60 mil becas para estudios en el extranjero otorgadas por Conacyt entre 1971 y 2015 (Conacyt, 2015b). Aunque los otros dos países también cuentan con programas de becas al extranjero, éstos históricamente han sido más pequeños. Sin embargo, los niveles de movilidad internacional podrían estar cambiando en el caso brasileño; el programa Ciencia sin Fronteras brasileña ha enviado a cerca de 100 mil estudiantes a cursar la maestría y el doctorado en el extranjero desde 2012 (Lloyd, 2017). A su vez, el sector de la investigación en México también es el más internacional de los tres países: $12 \%$ tuvo otra nacionalidad al nacer, mientras que en Brasil y en Argentina fue de $2 \%$.

En cuanto a los sueldos de los investigadores, los mexicanos reportaron ser los mejor pagados: ganaban el equivalente de USD\$43 000 al año, contra USD\$35 000 en Argentina y USD\$34 100 en Brasil (tales cifras fluctúan de forma marcada, según el tipo de cambio en cada país en un momento dado). Por otro lado, los brasileños reportaron ser los más estresados. En respuesta a la pregunta sobre si su trabajo era una "fuente de estrés personal", 38\% de los investigadores en Brasil estuvo en fuerte acuerdo o acuerdo, comparado con $27 \%$ en Argentina y $26 \%$ en México.

Tal diferencia podría obedecer al hecho de que Brasil fue de los primeros países latinoamericanos en implementar sistemas de evaluación en la educa- 
ción superior desde 1976; aunque el país no cuenta con un programa comparable al del SNI, sí exige cuotas de productividad a sus profesores-investigadores como requisito para acceder a mayores niveles (e ingresos) dentro de las universidades públicas (Lloyd, 2013). El hecho de que más de la cuarta parte de los investigadores en México reporte sufrir de estrés personal, y casi uno de cuatro de los brasileños, es un dato relevante, ya que manifiesta uno de los efectos no deseados de las políticas de pago por mérito y de las políticas de evaluación en general.

\section{La encuesta del SNI}

Esta breve exploración de los datos de la encuesta Changing Academic Profession da cuenta de algunos rasgos generales del sector de la investigación en México en los contextos nacional e internacional. Sin embargo, la segunda encuesta enfocada a los miembros del SNI permite analizar este ámbito con base en una muestra mucho mayor ( $n=5426)$.

\section{Perfil personal y académico}

Según los datos de 2008, los miembros del sNi se dividieron en $66 \%$ hombres y $34 \%$ mujeres. La edad promedio de los investigadores en ese año fue de 47 años, con un rango de edad de 28 a 86. De ellos, 89.5\% nació mexicano, los demás provinieron de otros 42 países, liderados por España (1.3\%), Cuba (1.1\%), Argentina (0.9\%), Rusia (0.9\%) y Estados Unidos (0.8\%). El predominio de los primeros países tiene sus razones históricas. El gobierno mexicano tiene una larga data de dar asilo a los exiliados de guerras civiles y dictaduras en América Latina y Europa; tales exiliados también han formado o poblado a importantes centros de investigación en el país, como fue el caso del Colegio de México y el Cinvestav (Didou y Gérard, 2010). Entre los que formaban parte del SNI, $4 \%$ con el tiempo adoptó la nacionalidad mexicana, por $94 \%$ que reportó contar con la misma en 2008 .

Un $95 \%$ contaba con un doctorado y $22.5 \%$ con un posdoctorado, niveles casi iguales a los identificados en la muestra RPAM-CAP para el subgrupo del SNI. En cuanto a sus niveles dentro del SNI, se divide como sigue: candidato (21\%), Nivel I (58.1\%), Nivel II (15.8\%) y Nivel III (5\%) (no se preguntó sobre el nivel de emérito, ya que son muy pocos). 
Figura 2

División de miembros del sNI por nivel

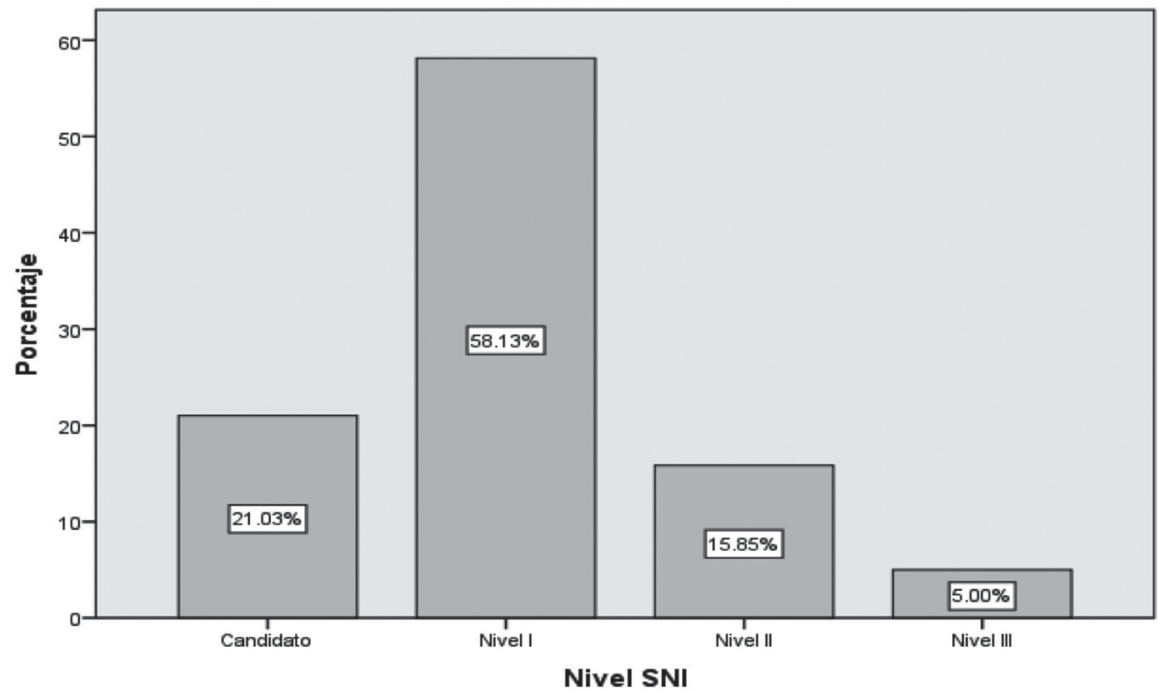

Casos ponderados por Ponderador SNI por género, nivel y área, muestra

Fuentes: Base RPAM-SNI 2007-2008.

Otro factor clave en caracterizar a este grupo es su nivel de capital cultural. Un $23 \%$ reportó tener padres que habían estudiado hasta la licenciatura y $11 \%$ contaba con estudios de posgrado, para un total de $34 \%$ que había concluido el nivel superior. Tal proporción es mucho mayor a la del promedio de la población mexicana; en 2015, 17\% de los mexicanos de entre 25 y 64 años contaban con un título universitario y apenas $1 \%$ había completado algún posgrado (OCDE, 2017). Cabe resaltar que los investigadores reportaron niveles de estudio más bajos para sus madres, con 5 y $10.5 \%$ alcanzando los grados de licenciatura y posgrado, respectivamente, no obstante, se espera que tal escenario cambie en los próximos años, debido al fuerte incremento de las mujeres en las universidades del país en las últimas décadas (De Garay y Del Valle, 2012).

\section{Movilidad internacional}

Para el periodo de la muestra, 9 de cada 10 miembros del sNI residía en México (89\%), mientras que 1\% vivía en Estados Unidos. El otro 8\% estaba distribuido a través de 30 países, incluyendo algunos menos esperados, como Líbano, Jordania y Nueva Zelanda. No obstante, gran parte de los que radi- 
caban en México tenía atrás una historia de movilidad nacional e internacional, sobre todo a nivel posgrado.

A nivel maestría, 79\% realizó sus estudios en México y 20\% en el extranjero. Entre los mayores países receptores fueron Estados Unidos (7.5\%), Francia $(3 \%)$ y Reino Unido (2.5\%). Entre los que contaban con doctorado (N=5 001), un menor porcentaje lo realizó en México (62\%). A su vez, 11.3\% estudió en Estados Unidos, 6.6\% en Francia, 6.3\% en España, 5\% en el Reino Unido, y los demás fueron esparcidos a través de otros 32 países. Resalta la fuerte diversidad de los países receptores, aunque se percibe una preferencia para los países anglosajones y europeos, que representaban 22 de los 37 países receptores. Llama la atención el hecho de que, por el mayor tamaño de la muestra, aparecen más países receptores que en la encuesta CAP-RPAM, donde sólo se registraron 19.

Figura 3

Países receptores de doctorantes

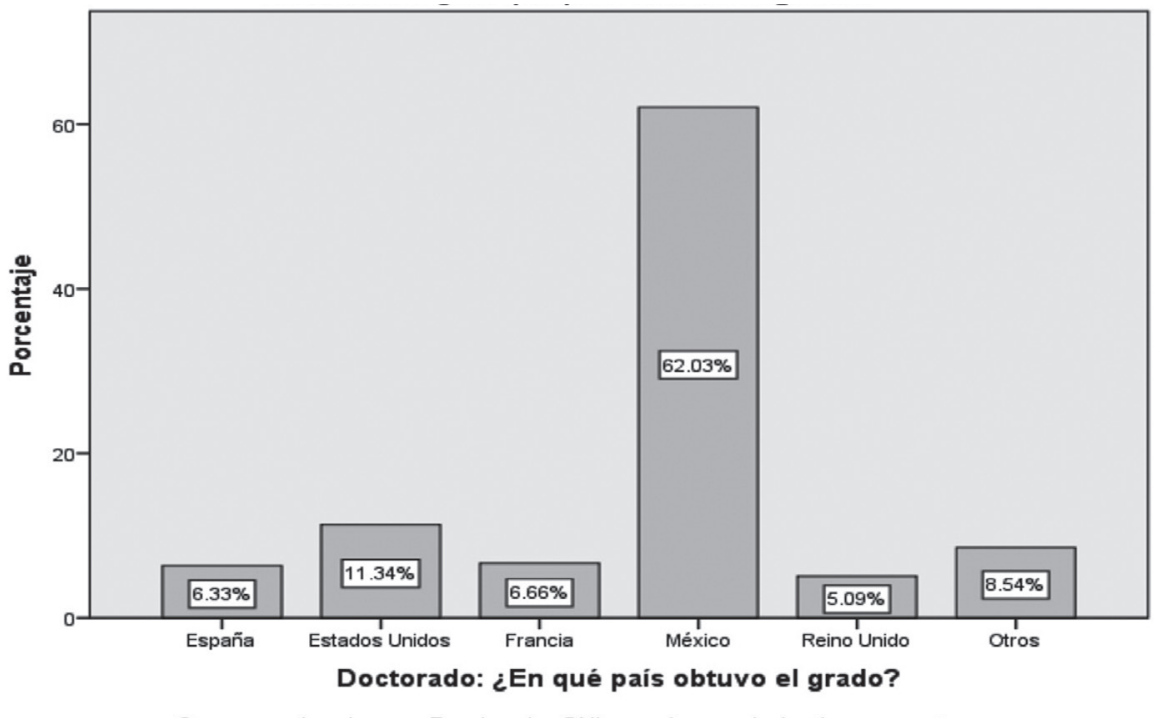

Casos ponderados por Ponderador SNI por género, nivel y área, muestra

Fuentes: Base RPAM-SNI 2007-2008.

En cuanto a quienes realizaron un posdoctorado ( $\mathrm{N}=1$ 197), el panorama geográfico cambia marcadamente. En total, 74\% emprendió sus estudios en el extranjero, contra 26\% que permaneció en México. Entre los principales países receptores estaban Estados Unidos con 36\%, seguido muy de lejos por España (6.6\%), Francia (5.7\%), Canadá (5.4\%), Reino Unido y Alemania, ambos con $5 \%$. La preponderancia de los estudios de posdoctorado en el extranjero, así como el relativamente alto porcentaje de investigadores que estudiaron el 
doctorado afuera reflejan uno de los sesgos del mercado académico en México: da más valor un título extranjero que uno local (Gérard y Grediaga, 2009).

Figura 4

Países receptores de posdoctorantes



PostDoctorado: ¿En qué país obtuvo el grado?

Casos ponderados por Ponderador SNI por género, nivel y área, muestra

Fuentes: Base RPAM-SNI 2007-2008.

\section{Condiciones y trayectorias laborales}

En el momento de la encuesta, 98\% de los entrevistados reportó ser de tiempo completo, un altísimo porcentaje si se considera que, en 2008, sólo 26\% de los académicos en México contaba con contratos laborales de este tipo, y para 2016 el porcentaje bajó a 22\% (EXECUM, 2018). La vasta mayoría de los miembros del SNI se ubicó en instituciones del sector público: 99\%, contra sólo $1.3 \%$ que reportó laborar en el privado. Entre ellos, 32\% trabajaba en una universidad pública estatal, $31 \%$ en una universidad federal y $16 \%$ en un centro de investigación pública. Esta situación seguramente obedece a las anteriores reglas del SNI, que sólo cubría las becas de los investigadores del terreno público. Tal regla cambió en 2008, cuando se facultó a Conacyt a cubrir la tercera parte de las becas para miembros del SNI en el ámbito privado, y después, en 2014, cuando se anunció que se cubriría la totalidad de las becas para el sector (Alcántara, 2014). Los cambios buscan estimular un crecimiento en la incorporación de investigadores de las instituciones priva- 
das, propósito que, sin embargo, no se ha concretado. Entre 2007 y 2015, el porcentaje de los miembros del sistema que provenía de las IEs privadas bajó de 4.2 a 3.9\%, aunque el número de individuos de ese grupo creció de 569 a 775 (EXECUM, 2017).

En términos de estabilidad laboral, 70\% contaba con un contrato permanente con definitividad (el equivalente a tenure en el sistema estadounidense), $10.6 \%$ con un contrato continuo sin duración específica, mientras $13.5 \%$ tenía un contrato por tiempo definido y $4.3 \%$ no tenía perspectiva de definitividad. Es decir, a diferencia del $66 \%$ de los profesores mexicanos que trabajaban por horas en 2008 (EXECUM, 2018), más del 80\% de los miembros del SNI tenía una seguridad laboral en el mediano o largo plazo. En cuanto al nivel de su contrato actual, $77 \%$ reportó ser titular, $13 \%$ asociado y $0.9 \%$ asistente.

Quizás el mayor indicador del estatus privilegiado de los miembros del sistema es su nivel económico; como se ha mencionado, éstos gozan de sueldos muy por encima del promedio de los académicos mexicanos, debido a una combinación de estímulos del propio programa y de sus instituciones, entre otros factores. El ingreso promedio reportado fue de USD\$44 334 al mes, lo que representó el doble del ingreso mensual bruto: de \$21 298. Aunque los estímulos de productividad (y antigüedad) de las instituciones fueron un factor importante, generando un promedio de \$8 962 al mes, fueron menores que los incentivos externos (que consiste en su mayoría del SNI), que fue de \$11 595. Tal brecha es considerablemente mayor que la reportada en la encuesta RPAM-CAP, por lo que la segunda encuesta permite ver con más claridad la magnitud de los beneficios económicos que otorga el SNI a sus integrantes.

Tabla 2

Distribución de ingresos (promedio) en USD

\begin{tabular}{ll}
\hline Tipo de ingreso (bruto) & Monto mensual \\
\hline Contrato institucional & $\$ 21298$ \\
\hline Sistema institucional de incentivos & $\$ 8962$ \\
\hline Incentivos externos & $\$ 11595$ \\
\hline Total $^{*}$ & $\$ 44334$ \\
\hline * Total no es igual a los demás montos, porque son respuestas a distintas preguntas. \\
Fuente: elaboración propia con base en la Base RPAM-SNI 2007-2008.
\end{tabular}

Como es de esperarse, hay una fuerte correlación entre el nivel del sNi y los ingresos reportados por sus miembros. Por ejemplo, los candidatos percibían un promedio (en números redondos) de \$31 300 al mes, los de Nivel I, \$43 700, Nivel II, \$54 800, y Nivel III, \$71 100. Tales beneficios se terminan, sin embar- 
go, a la hora de jubilarse. A la vez, en una mayoría de universidades del país, los académicos jubilados reciben una pequeña proporción de su sueldo al retirarse, una política que fomenta que muchos de ellos trabajan hasta muy avanzada edad o hasta morirse (Lloyd, 2013).

\section{Movilidad laboral}

Un gran porcentaje de los miembros del SNI comenzó a trabajar antes de finalizar su doctorado: $34 \%$ tuvo un contrato durante sus estudios de ese nivel. También 86\% dijo haber trabajado en IEs desde la obtención del primer grado; entre éstos, $14 \%$ había trabajado en el sector privado. También se aprecia un nivel de movilidad relativamente baja, pues 52\% había trabajado en una o dos universidades desde la obtención del primer grado, mientras una pequeña proporción $2.7 \%$ había trabajado en cinco instituciones distintas. Desde la obtención del doctorado hay mayor estabilidad: 36\% reportó mantenerse en la misma institución, mientras que otro $32 \%$ sólo cambió a una institución adicional.

Entre el sector de centros e instituciones de investigación hay aún menor movilidad: $57 \%$ se mantuvo en la misma institución desde la obtención del primer grado y $25 \%$ sólo había trabajado en dos instituciones. A su vez, desde la obtención del último grado, $68 \%$ de los investigadores de los centros se quedó en la misma institución y $18 \%$ pasó a otra. Tal situación refleja el grado de seguridad laboral de que gozan los miembros del sNI comparado con los demás académicos en México; a su vez, los investigadores de los centros de investigación parecen ser un grupo aún más privilegiado, ya que una mayoría de las plazas en esas instituciones son de tiempo completo.

Curiosamente, la encuesta no preguntó si hubo un cambio de trabajo reciente, sino si hubo un esfuerzo en ese sentido. Aun así, sólo $7 \%$ reportó "tomar acciones" en los últimos cinco años para realizar un "cambio importante en su trabajo". Cuando se preguntó si tomó "acciones para cambios potenciales", 26\% respondió que sí. A su vez, 11\% reportó tomar acciones para cambiar de puesto académico en otra universidad o IES en el país, y 6.6\% para cambiarse a un puesto en el extranjero.

Visto en números absolutos (352) de ese último grupo, no es cosa menor que tantos profesores buscaran trabajo en otro país. A su vez, 13.5\% reportó dar un curso en el extranjero durante el año académico vigente o previo, sobre todo en Estados Unidos (2.4\% de la muestra) y España (1.9\%). No obstante, cuando fueron cuestionados sobre los apoyos para movilidad interna- 
cional, 73\% opinó que fueron "muy pobres" o "pobres". De igual forma, 59\% opinó lo mismo sobre los apoyos para la movilidad nacional.

\section{Trabajo de investigación}

En promedio, los encuestados reportaron dedicar 21.6 horas a la investigación (es decir, poco más de la mitad de su contrato laboral), contra sólo siete horas a la docencia. No obstante, aunque $67 \%$ describió esta labor como la primera en importancia, $21 \%$ dijo que era su segunda prioridad, un dato algo sorprendente dado que el sistema busca fomentar a la investigación científica como labor primordial entre sus integrantes. En lo que respecta al nivel de productividad, sólo $26 \%$ reportó haber producido un libro de su autoría o coautoría en los últimos tres años, 5.6\% informó concluir tres o cuatro y 1.3\% cinco o seis. La producción de artículos también fue relativamente baja: $22 \%$ reportó producir uno o dos artículos, $28 \%$ dos, y $18 \%$ de tres a cuatro.

En términos del nivel de colaboración en investigación, 79\% reportó colaborar con colegas en otras IES mexicanas y $62 \%$ con sus pares en el extranjero. A su vez, sólo $40 \%$ formaba parte de un cuerpo académico Promep, el programa que fomenta la formación de redes de investigación. Esta baja participación probablemente obedece a que el programa está dirigido hacia las universidades estatales, y no participan los integrantes de la UNAM, el Cinvestav o los otros grandes centros de producción científica en el país. Sin embargo, los encuestados tampoco evaluaron bien los apoyos institucionales para la investigación, que fueron calificados como "muy pobres" o "pobres" por $67 \%$ de los encuestados.

\section{Diferencias de género}

Mientras las cifras anteriores dan un panorama global de los miembros del SNI, no dejan apreciar las fuertes diferencias entre los distintos subgrupos: sobre todo entre hombres y mujeres. Varios investigadores (por ejemplo, Didou y Gérard, 2010; 2011) han insistido en los fuertes sesgos de género, que dificultan las trayectorias laborales de las mujeres investigadoras. Estas diferencias, que obedecen a que las mujeres tienen que compaginar sus responsabilidades familiares y académicas en mayor grado que los hombres, son reflejadas en la baja presencia de las mujeres dentro del SNI y, sobre todo, en los más altos niveles. Por ejemplo, 13.5\% de las mujeres reportó haber interrumpido su trabajo para cuidar a niños o adultos mayores, y $20 \%$ opinó 
que tal situación tuvo un efecto negativo en su trabajo académico. Entre los hombres, $3.5 \%$ interrumpió su trabajo y $8 \%$ reportó que sufrió un impacto negativo.

Según Didou y Gérard (2011), mujeres y hombres compiten y participan en la producción científica en "condiciones de desigualdad" (p. 33). Mientras $77 \%$ de los hombres se ubicaron en los primeros dos niveles del SNI (candidato y Nivel 1), 84\% de las mujeres estaban en esos niveles. En contraste, la proporción de hombres que alcanzaron el Nivel III fue más del doble que la de las mujeres: 6.2 contra 2.7\%. Al analizar los datos de 2009, a 25 años de la creación del sistema, Didou y Gérard (2009) concluyeron lo siguiente: “Es indudable que ser mujer funciona todavía como un factor discriminante en cuanto a esquemas y temporalidades para los recorridos en el SNI, tanto en lo que se refiere a oportunidades de ascenso como a plazos de carrera" (p. 34).

A su vez, las mujeres tardaban más en subir por los peldaños del sistema, lo que tuvo un impacto negativo en sus ingresos. Mientras los hombres del máximo nivel del sNi percibían $\$ 71700$ al mes, las mujeres en ese nivel (una pequeña minoría) ganaban $\$ 68$ 800. Las diferencias se reflejaban en todos los niveles, como se percibe en la Tabla 3.

Tabla 3

Promedio de ingresos, por género y nivel del SNI (USD)

\begin{tabular}{l|cc|cc}
\hline & \multicolumn{2}{|c|}{ Mujeres } & \multicolumn{2}{c}{ Hombres } \\
\hline Nivel & Ingreso mensual & Número & Ingreso mensual & Número \\
\hline Candidato & $\$ 30002$ & 363 & $\$ 32476$ & 602 \\
\hline I & $\$ 41543$ & 962 & $\$ 44695$ & 1925 \\
\hline II & $\$ 50496$ & 225 & $\$ 56846$ & 571 \\
\hline III & $\$ 68818$ & 43 & $\$ 71743$ & 196 \\
\hline Promedio & $\$ 40921$ & 1595 & $\$ 46177$ & 3294 \\
\hline
\end{tabular}

Fuente: Elaboración propia con base en los datos de la encuesta RPAM-SNI 2007-2008.

\section{La evolución del sistema: 2008-2015}

La encuesta aplicada a los miembros del SNI da un panorama amplio y detallado sobre los integrantes del sector, de lo cual se hizo una selección reducida de datos para los fines de este artículo. Como la información data de hace una década, vale la pena revisar la más reciente sobre el sector para constatar posibles cambios y tendencias. En general, los pocos datos disponibles para el periodo reciente no reflejan grandes cambios, aunque se ha ampliado la 
membresía del sistema de forma marcada durante la última década (Conacyt, 2015a). El Informe General del Estado de la Ciencia, la Tecnología y la Innovación, de 354 páginas, le dedica una sección de 6 páginas al SNI, en donde provee información sociodemográfica acerca de sus integrantes.

En 2015, hubo 23316 miembros, comparado con 14681 en 2008, un incremento de $59 \%$ en sólo 8 años. Durante el mismo periodo, el presupuesto del Conacyt para el programa subió casi 100\%, de 2080 millones de pesos a 3992 millones (sin ajustar por la inflación). A la vez, hubo cambios menores en el perfil de los investigadores. El porcentaje de mujeres subió ligeramente: de $32 \%$ a $36 \%$. Sin embargo, permaneció la alta concentración de miembros en la capital del país: 34\%. En este sentido, destaca la UNAM, con 18\% del total de miembros en 2015, seguido por el Instituto Politécnico Nacional y la Universidad Autónoma Metropolitana, con 5\% cada una (Conacyt, 2015a).

\section{Conclusiones}

Las dos encuestas aplicadas por la Red de Investigadores sobre Académicos en México dan un panorama muy amplio sobre características, trayectorias y niveles de movilidad de los investigadores en México a finales de la primera década del siglo xxi. Los datos oficiales y las investigaciones más recientes permiten esbozar el perfil de este sector en México y compararlo con otros países. En general, el resultado da una imagen mixta. Mientras se ha construido un grupo de investigadores con altos niveles de estudios y experiencia internacional, éste sigue representando una pequeña mayoría entre los académicos del país. Los miembros del Sistema Nacional de Investigadores - el núcleo del sector - representaban sólo $5 \%$ de los 400 mil académicos que laboraban en el país en 2015 (EXECUM, 2018).

A su vez, representan una minoría privilegiada. Según las encuestas, ganaban en promedio el doble de lo que percibían sus pares sin membresía, aun cuando estos últimos fueron profesores de tiempo completo (por sí sólo, otra minoría privilegiada). Los SNI también fueron más educados - casi todos contaban con el nivel de doctorado, y la quinta parte con posdoctorado - y ostentaban mayores niveles de capital cultural, de experiencia y de movilidad internacional. Por último, estaban concentrados en los polos de mayor influencia y desarrollo socioeconómico del país. Es decir, pertenecían a un círculo virtuoso.

No obstante, el sistema de investigación en México enfrenta serios retos y tensiones. El primero de ellos es la desigualdad de oportunidades entre hombres y mujeres, con las segundas en una posición de franca desventaja. 
Asimismo, a pesar de que los miembros del SNI perciben mayores sueldos que los demás académicos, no gozan de condiciones comparables con los de sus pares en Estados Unidos, Canadá o algunos países de Europa; por eso deviene la gran migración hacia esos polos de investigación. A su vez, los investigadores reportaban sufrir de altos niveles de estrés laboral, en gran medida debido a la cultura de "publicar o morir" y a las demandas del Estado evaluador.

Algunos de estos problemas son generales a nivel nacional. Por ejemplo, la fuerte concentración de los académicos en pocos centros de investigación e IES y la poca movilidad entre instituciones; no existen condiciones y contratos comparables entre instituciones públicas a nivel nacional, como sí existen en Brasil, por ejemplo (Lloyd, 2013). A su vez, debido a la falta de un adecuado sistema de jubilación en la mayoría de las IES, muchos investigadores y profesores permanecen en sus trabajos hasta una edad muy avanzada, o incluso hasta morir. Los miembros del SNI, por ejemplo, dejan de percibir sus becas al momento de jubilarse, excepto en los escasos casos de los miembros eméritos. Tal situación no sólo provoca incertidumbre y estrés personal, sino que impide la entrada de nuevos investigadores al sistema.

Sin embargo, quizás el efecto más "perverso" del programa de pago por mérito por excelencia en México ha sido la estratificación de la academia entre una pequeña élite de investigadores y la vasta mayoría que se dedica a la docencia, y cuyas condiciones laborales y económicas suelen ser muy inferiores. Quizá tal desenlace sería justificable si el sistema hubiera logrado un gran incremento en la productividad científica en el país. No parece ser el caso.

México sigue estando muy a la zaga de Brasil en cuanto al número de artículos publicados en revistas indexadas, así como en la producción de patentes y otros indicadores. Entre 2009 y 2016, el número de artículos producidos por investigadores en México y registrados en el Web of Science aumentó de 9 307 a 16 228. Sin embargo, durante el mismo periodo en Brasil, que no cuenta con un programa de pago por mérito para investigación, el número de artículos en el índice internacional creció de 30921 a 53004 (Web of Science, 2018). Es decir, Brasil, un país cuya población es menos del doble que la de México, produce 3.2 veces el número de artículos indexados que México.

Tal situación puede obedecer en parte al hecho de que el gasto total en CyT en México se ha mantenido prácticamente igual durante las últimas décadas, entre 0.38 y $0.5 \%$ del PIB, a pesar de leyes y de programas que mandatan un mínimo de 1\% en el Gasto de Investigación Científica y Desarrollo Experimental (GIDE). En comparación, la inversión brasileña es varias veces más grande: $1.28 \%$ del PIB. 
Tales resultados han llevado a algunos especialistas a concluir que las políticas de ciencia y tecnología adoptadas por México durante las últimas décadas son "una mala copia" de las empleadas por los países desarrollados (Galaz y Gil, 2009). Como mínimo, tales políticas han sido insuficientes para el desarrollo de una economía basada en el conocimiento, con implicaciones no sólo para los propios investigadores sino para la sociedad en su conjunto.

\section{Referencias}

Alcántara Santuario, Armando (2014, 30 de enero) ¿Subsidio público a universidades privadas? Campus Milenio.

Balbachevksy, Elizabeth (2015). The Latin American university model and the challenges posed by the reforms: Perspectives from the academics. En W. K. Cummings y U. Teichler (eds.), The relevance of academic work in comparative perspective, The changing academy - The Changing Academic Profession in international comparative perspective 13, (pp. 239-252). Switzerland: Springer International Publishing.

Bernheim, Carlos T., y Chaui, Marilena d. S. (2003). Challenges of the university in the knowledge society, five years after the World Conference on Higher Education. unESCO Forum Occasional Paper Series, 4. Consultado en http://unesdoc. unesco.org/images/0013/001344/134422e.pdf.

Bourdieu, Pierre (1986). The forms of capital. En J. G. Richardson (Ed.), Handbook of theory and research for the sociology of education, (pp. 83-93). Nueva York: Greenwood.

Buendía, Angélica, García Salord, Susana, Grediaga, Rocío, Landesman, Monique, Rodríguez-Gómez, Roberto, Rondero, Norma... y Vera, Héctor (2017, 5 de julio). Queremos evaluar y terminamos contando: Alternativas para la evaluación del trabajo académico. Nexos. Consultado en https://educacion. nexos.com.mx/?p=588

Carreón Mendoza, Héctor, y Melgoza Ramos, Ricardo (2012). México hacia una sociedad del conocimiento. Nóesis, 21 (41), 121-135.

Chavoya Peña, María L. (2013). Ser investigador: la zanahoria de los doctores recién egresados en México. Estudio de un caso. Diálogos sobre Educación, 4 (6). Disponible en http://www.revistascientificas.udg.mx/index.php/DSE/ article/view/3722/3498

Christensen, Finn, y Manley, James (2011). The Allocation of Merit Pay in Academia: A Case Study. Economics Bulletin, 31(2): 1548-1562.

Consejo Nacional de Ciencia y Tecnología (Conacyt) (2014). Programa Especial de Ciencia, Tecnología e Innovación 2014-2018. Consultado en http:/ /www. siicyt.gob.mx/index.php/normatividad/nacional/631-3-programa-especialde-ciencia-tecnologia-e-innovacion-2014-2018/ file

Conacyt (2015a). Informe general del estado de la ciencia, la tecnología y la innovación. México 2015. México: Conacyt. Consultado en http://www.siicyt.gob.mx/ index.php/transparencia/informes-conacyt/informe-general-del-estado- 
de-la-ciencia-tecnologia-e-innovacion/informe-general-2014/1572-informegeneral-2014/file

Conacyt (2015b) Diagnóstico del Programa Presupuestario de Becas de Posgrado y Apoyos a la Calidad (S190). Dirección Adjunta de Posgrado y Becas. Consultado en http://www.transparenciapresupuestaria.gob.mx/work/models/PTP/Reingenieria_Gasto/imagenes/Ventanas/Ramo_38/38S190.pdf

Conacyt (2018). Padrón de beneficiarios apoyos infraestructura científica. Consultado en https://www.conacyt.gob.mx/index.php/sni/convocatorias-conacyt/convocatorias-apoyos-complementarios/padron

Canales, Alejandro (2007). La política científica y tecnológica en México: el impulso contingente en el periodo 1982-006. México D.F.: Flacso México.

Covarrubias Papahiu, Patricia (2017). Situación Actual de los Programas de Evaluación Académica de la Educación Superior Mexicana: Sus Efectos en el Trabajo Académico. Revista Iberoamericana de Evaluación Educativa, 2017, 10 (2), 187-209

De Garay, Adrián, y Del Valle Díaz-Muñoz, Garbriela V. (2012). Una mirada a la presencia de las mujeres en la educación superior de México. Revista Iberoamericana de Educación Superior, 3 (6).

Didou Aupetit, Sylvie, y Gérard, Etienne (2010). El Sistema Nacional de Investigadores, veinticinco años después. La comunidad científica, entre distinción e internacionalización. México: ANUIEs.

Didou Aupetit, Sylvie, y Gérard, Etienne (2011). El Sistema Nacional de Investigadores en 2009 ¿Un vector para la internacionalización de las élites científicas? Perfiles Educativos, 33 (132), 29-47.

Escobar Latapí, Agustín, y Martin, Susan (Coords.) (2006). La gestión migratoria México-Estados Unidos: Un enfoque binacional. Guadalajara: CIESAs Occidente, Universidad de Georgetown.

Explorador del Estudio Comparativo de Universidades Mexicanas (EXECUM) (2017/2018). Consultado en http:/ / www.execum.unam.mx/

Galaz Fontes, Jesús F., y Gil Antón, Manuel (2009). La profesión académica en México: Un oficio en proceso de reconfiguración (2009). Revista Electrónica de Investigación Educativa, 11 (2).

Galaz Fontes, Jesús F., Padilla González, Laura E., Gil Antón, Manuel, Martínez Stack, Jorge G., y Jiménez Loza, Leonardo (2009). La Reconfiguración de la Profesión Académica en México: Nota metodológica. Ponencia presentada al seminario internacional El futuro de la profesión académica: Retos para los países emergentes, en Buenos Aires, 30 de marzo-1 de abril.

Galaz Fontes, Jesús F., De la Cruz Santana, Ana L., Rodríguez García, Rocío. (2010). El Académico Mexicano Miembro del Sistema Nacional de Investigadores: Una exploración inicial de su trayectoria profesional. Ponencia presentada en el I Congreso de los Miembros del Sistema Nacional de Investigadores, mayo 5-8, Querétaro, México.

Galaz Fontes, Jesús F., Martínez Stack, Jorge G., Estévez Nénninger, Etty H., De la Cruz Santana, Ana L., Padilla González, Laura E., y Gil Antón, Manuel (2012). Los divergentes mundos de la docencia y la investigación entre los académicos mexicanos: Tendencias e implicaciones. En J. F. Galaz Fontes, M. 
Gil Antón, L. E. Padilla González, J.J. Sevilla García, J. J. Arcos Vega y J. G. Martínez Stack (Coords.), La reconfiguración de la profesión académica en México, (pp. 129-155). Culiacán, México: Universidad Autónoma de Sinaloa, Universidad Autónoma de Baja California.

Galaz Fontes José F. y Gil Antón, Manuel (2013). The impact of merit-pay systems on the work and attitudes of Mexican academics. Higher Education, 66 (3), 357-374.

García Salord, Susana (2000). La carrera académica: Escalera de posiciones y laberinto de oportunidades. Ponencia presentada en el Encuentro de Especialistas en Educación Superior, México D.F.: Centro de Investigaciones en Ciencias y Humanidades-unAM.

García Salord, Susana (2001). La simulación: El fantasma que recorre a la vida académica cotidiana. Universidad y Sociedad, 1 (1), 97-103

Gérard, Etienne, y Grediaga Kuri, Rocío (2009). ¿Endogamia o exogamia científica? La formación en el extranjero, una fuerte influencia en las prácticas y redes científicas, en particular en las ciencias duras. En Didou-Aupetit, S. (Ed.), Gérard, E. (Eds.). Fuga de cerebros, movilidad académica y redes científicas, (pp. 137-160). México: ISEALC-Cinvestav-IRD.

Gil Antón, Manuel (2008). Los académicos en instituciones privadas que captan demanda. Revista Mexicana de Investigación Educativa, 37 (1), 115-121.

Gobierno de México (2017). Programa para el Desarrollo Profesional Docente, para el Tipo Superior (Prodep). Consultado en http://www.dgesu.ses.sep. gob.mx/PRODEP.htm

Field, Laura (2015) Using Outperformance Pay to Motivate Academics: Insiders' Accounts of Promises and Problems. Australian Universities' Review, 57 (2) 5-16.

Izquierdo Sánchez, Miguel A. (1998). Políticas y experiencias de evaluación de académicos. Revista Colección Universitaria, 30 (jul-dic.).

Lloyd, Marion (2013). Las políticas de fomento a la ciencia y tecnología en México y Brasil: Un estudio de caso de la Universidad Nacional Autónoma de México y la Universidad de São Paulo. Tesis de Maestría en Estudios Latinoamericanos, Universidad Nacional Autónoma de México.

Lloyd, Marion (2017). Equidad versus mérito en la universidad: Las politicas de acción afirmativa en Brasil. Tesis de Doctorado en Ciencias Políticas y Sociales, Universidad Autónoma Nacional de México.

Marginson, Simon, y Ordorika, Imanol (2010). Hegemonía en la era del conocimiento: competencia global en la educación superior y la investigación científica. México: SES/UNAM.

Marmolejo, Francisco (2009). Redes, movilidad académica y fuga de cerebros en América del Norte: el caso de los académicos mexicanos, (pp. 101-116). En S. Didou-Aupetit, E. Gérard (Eds.). Fuga de cerebros, movilidad académica y redes científicas, (pp. 137-160). México: ISEALC-Cinvestav-IRD.

Martínez Stack, Jorge, Lloyd, Marion, y Ordorika, Imanol (2015). The impact of government policies on the profiles and attitudes of academics in two emerging economies: Brazil and Mexico. En W. K. Cummings y U. Teichler (Eds.), The relevance of academic work in comparative perspective, The changing academy- 
The Changing Academic Profession in international comparative perspective 13, (pp. 193-215). Switzerland: Springer International Publishing.

McCrea, Elizabeth, y Deyrup, Martha (2016). The devil is in the details: A review of merit pay in higher education. Ponencia en el decimosexto Congreso de la Eastern Management Academy, Universidad de Yale, New Haven, Connecticut, 4-7 de mayo.

Minasyan, Eva T.; Midova, Venera O., Danko, Olga A., Balakhanova, Dariko K. (2017). Implementing New Performance Pay-Based Schemes in Higher Educational Institutions. European Journal of Contemporary Education, 6 (4), 748-756.

Olivares Alonso, Emir (2014, 8 de julio). Anuncia Conacyt estrategia para crear red de la ciencia mexicana. La Jornada. Consultado en http://www.jornada. unam.mx/2014/07/08/sociedad/037n1soc

Ordorika, Imanol, y Navarro, Miguel A. (2006). La investigación académica y las políticas públicas en la educación superior; el caso mexicano de pagos por méritos. Revista del Centro de Estudios y Documentos sobre la Educación Superior Puertorriqueña (CEDESP), 1, 53-72.

Ordorika, Imanol, y Lloyd, Marion (2014). International rankings and the contest for university hegemony. Journal of Education Policy, 30 (3), 385-405.

Organización para la Cooperación y Desarrollo Económicos (OCDE) (2017). Panorama de la educación 2017. Consultado en http:/ / www.oecd.org/education/ skills-beyond-school/EAG2017CN-Mexico-Spanish.pdf

Rodríguez, Roberto (2017, 6 de febrero). Reforma del sni. Educación Futura. Consultado en http:/ / www.educacionfutura.org/reforma-del-sni/

Red de Indicadores de Ciencia y Tecnología Iberoamericana e Interamericana (RICYT) (2017/2018). Consultado en http:/ / www.ricyt.org/indicadores

Red de Investigadores sobre Académicos (RDISA). Base de datos de la encuesta $L a$ Reconfiguración de la Profesión Académica en México (RPAM).

Red de Investigadores sobre Académicos (RDISA). Base de datos de la encuesta sobre miembros del Sistema Nacional de Investigadores (SNI) en México.

Ruíz Jaimes, Elizabeth (2014, 14 de abril). Repatriación de talento, programa insignia del Conacyt. El Economista.

Terpstra, David E., y Honoree, Andre L. (2009). Merit pay plans in higher education institutions: Characteristics and effects. Personal Public Management, 38 (4), 55-77.

Tuirán, Rodolfo, y Ávila, José L. (2013, 6 de enero). ¿De la fuga a la circulación de talentos? Revista Este País.

Web of Science. Base de datos. Consultado en http://apps.webofknowledge. com/WOS_GeneralSearch_input.do?product=WOS\&search_mode=General Search\&SID=5Cpmljv81xyyes69zzl\&preferencesSaved $=$ 
